\title{
APLICACIÓN DIDÁCTICA DE LOS MAPAS Y LA CARTOGRAFÍA COMO INSTRUMENTOS DE FORMACIÓN EN EL AULA
}

\section{DIDACTIC APPLICATION OF THE MAPS AND THE CARTOGRAPHY AS TRAINING TOOLS IN THE CLASSROOM}

\author{
Eva Ortiz Cermeño*
}

RESUMEN

El objetivo principal del presente artículo consiste en exponer las apreciaciones y los procederes del estudiantado en el manejo de la cartografía, concretamente con los mapas histórico-geográficos en las clases de Historia Contemporánea en la Región de Murcia (España). La metodología empleada cualitativa y descriptiva se centra en las historias de vida del discente. Los resultados indican una visión pedagógica positiva del uso de estos medios para asimilar el aprendizaje adquirido.

PALABRAS CLAVE: CARTOGRAFÍA * HISTORIA * GEOGRAFÍA * MÉTODO DE APRENDIZAJE * ENSEÑANZA

\section{ABSTRACT}

The main objective of this article is to expose the assessments and procedures of the students in the management of cartography, specifically with historical-geographical maps, in the Contemporary History classes in the Region of Murcia (Spain). The methodology used, qualitative and descriptive, centers on the life stories of the students. The results indicate a positive pedagogical vision of the use of these means to assimilate the acquired learning.

KEYWORDS: CARTOGRAPHY * HISTORY * GEOGRAPHY * LEARNING METHOD * TEACHING

Universidad de Murcia, España.

evaortiz@um.es 


\section{INTRODUCCIÓN}

De acuerdo con Parellada (2017), la interpretación y la elaboración de mapas a través de las representaciones gráficas proporcionan al educando la comprensión espacial de conceptos, fenómenos, procesos o acontecimientos producidos en el territorio. El material cartográfico (mapas y planos) utilizado como herramienta pedagógica en el aula establece un sistema de significados porque transmite, informa, representa, observa y examina un orden social (Harley, 2005).

La utilidad de los mapas como procedimiento programado desde la Ley Orgánica General del Sistema Educativo (LOGSE) (Jefatura del Estado, 1990) pretende conseguir conocimiento, hacer comprender al estudiantado cómo funcionan los espacios de las sociedades humanas, razonar geográficamente, pensar el espacio (Clary, 1995). Souto (1998) establece que la didáctica de la geografía plantea el restablecimiento del saber a partir de la interacción entre el docente y el estudiantado, los materiales que se aplican y el contexto socio-escolar. Para conseguir un aprendizaje significativo, los instrumentos cartográficos permiten innovar porque contribuyen a completar los contenidos y replantear los problemas de la ciencia (Andreozzi y Benedetti, 6-8 de octubre de 2005).

El Diccionario de la Real Academia Española (2016) define la cartografía como "arte de trazar mapas topográficos", así como, la "ciencia que los estudia". El término viene del griego chartis (mapa) y graphein (escrito). Por tanto, se puede definir como "el conjunto de ciencias, técnicas y artes cuyo fin es la representación gráfica y fiel del territorio, desde las menores superficies hasta la totalidad de la Tierra" (Martín, 1997, p. 4, como citado en Crespo y Fernández, 2011, p. 406).

Las diferentes probabilidades que representa la Geografía de la Percepción para el trabajo pedagógico en la enseñanza de la Cartografía, resultan útiles para la educación espacial, especialmente porque favorecen una aproximación distinta, individual y subjetiva del entorno que rodea a los escolares. Las percepciones instauran elementos en los que contribuyen, de manera holística, las impresiones sensoriales y otros elementos como la memoria, las representaciones mentales, las convicciones y los hábitos de las personas. De igual modo, se tienen en cuenta los conocimientos y las experiencias previas de los escolares acerca de los temas que se imparten para contribuir a afianzar el aprendizaje significativo. También se aprenden y se ponen en prácticas diferentes valores cívicos como el respeto hacia otros puntos de vista discordantes a los propios y la convivencia entre compañeros propiciando espacios de aula democráticos (Araya y Pacheco, 2008).

El mapa es el resultado de la necesidad del ser humano de averiguar la localización de hechos y objetos en la superficie terrestre, de ahí que apareciera la cartografía como ciencia $y$ disciplina especializada en su elaboración. De igual manera, el mapa es una herramienta fundamental en el quehacer geográfico, siendo la Geografía la ciencia que mejor uso hace de este lenguaje de comunicación, ya que lo comprende, analiza y elabora a partir de datos espaciales (Perea y Mayor, 2014). "Lo que hace que un mapa sea un mapa es su cualidad de representar una situación local; tal vez deberíamos llamarlo imagen de situación o sustituto situacional. La función principal de esa imagen es transmitir información situacional" (Buisseret, 2004, p. 16).

Las propiedades tangibles del mapa y su cualidad de transmisor de información, estimulan la capacidad de análisis del educando, haciéndole más accesible una realidad compleja (Hernando, 2000). Igualmente, un mapa motiva la atención sinóptica y la analítica, combina imagen y razonamiento, lo que se trascribe en "una lectura prácticamente instantánea que favorece la recepción en un tiempo mínimo de un máximo de información disponible" (Delgado, 2002, pp. 336-337).

Del mismo modo, los mapas son una fuente de información de primer orden (distancia, superficie, valores) para la investigación al infundir problemas y facilitar la correlación del espacio entre distintas variables. Además, los mapas permiten establecer explicaciones sobre aspectos físicos y sociales de una determinada área, lo cual permite al alumnado construir y elaborar su aprendizaje 
por descubrimiento de un modo integral y colectivo fomentando el pensamiento crítico y creativo (Carrera et al., 1993).

\section{LOS MAPAS COMO RECURSO DIDÁCTICO: REPRESENTACIÓN DEL ESPACIO}

De acuerdo con Quinquer (1997), "un método didáctico es una forma determinada de organizar las actividades pedagógicas con el propósito de conseguir que los estudiantes puedan asimilar nuevos conocimientos y puedan desarrollar capacidades o habilidades cognitivas" (p. 99). Algunos mapas interesantes son las cartas formadas por conchas sobre un enrejado de fibra de palma y que eran empleadas para la navegación, ubicados en las Islas Marshall (al noreste de Australia). Algunas réplicas de estos mapas se encuentran en diferentes museos de Inglaterra, España y Alemania (Raisz, 1985).

El estudio del mapa no es sólo una herramienta geográfica sino un lenguaje que toda persona educada debe de dominar puesto que es imprescindible para el hombre adulto $y$, por ese valor que su conocimiento tiene, el trabajo con el mapa y la manera de transmitir su lenguaje se ha convertido en una preocupación de los profesores de geografía en muchos países (Piñeiro, 2003, p. 350).

En consonancia con Ramaswamy (2001), las comunidades observan el tamaño, la extensión y los perímetros del territorio de la nación mediante el mapa. Este es un instrumento preciso que permite a la ciudadanía distinguir cómo y dónde están espacialmente ubicados, de lo contrario, el espacio nación seguiría siendo una entidad abstracta. Es factible que sea esta la razón, explica el autor, por lo que en cada escuela, pública o privada, de la mayoría de los países, siempre ha habido un mapa del Estadonación presente en el aula.

Siguiendo a Jerez (2006), se pueden constituir tres categorías en cuanto a los mapas: 1) Técnica: para conocer y entender el territorio y los distintos fenómenos geográficos que suceden, así como, la base de datos de información territorial y espacial. 2) Didáctica: su función principal es alfabetizar cartográficamente, enseñar y aprender a leer, interpretar y comprender el lenguaje cartográfico y construir significados a partir de este. 3) Educativa: su función es comunicar una realidad que permita su interpretación de forma crítica, con el fin de desarrollar capacidades intelectuales, procedimentales $y$ actitudinales para que el alumnado conozca el exterior en el que se desenvuelve, los fenómenos que le traban y sus relaciones con el medio.

De acuerdo con Luque (2011), los contenidos cartográficos y el trabajo con mapas son esenciales en el aprendizaje de las ciencias sociales $y$, particularmente, en la Geografía, que se ocupa de fenómenos tan diversos como el clima, el relieve, la vegetación o el hábitat. Representarlos sobre un mismo soporte proporciona un aprendizaje comprensivo. Del mismo modo, la expresión cartográfica habilita el análisis geográfico temático de diferentes ámbitos espaciales (locales, regionales, nacionales, etc.).

Para elaborar mapas en el aula hay que preparar con el estudiantado conceptos básicos de cartografía (puntos cardinales, orientación, utilización de convenciones), para que elaboren sus representaciones cartográficas desde sus impresiones, a través del pensamiento crítico, subjetivo y reflexivo. Por ejemplo, pueden representar la ruta de la casa a la escuela, este modelo es uno de los dispares tipos de mapas que se pueden construir a partir del uso de la cartografía social: "Los mapas que se pueden elaborar desde la escuela con la comunidad son de diversa índole, y su construcción debe partir de las necesidades de los mismas comunidades $y$ a los contextos socio-ambientales a las que se hallan" (Córdoba, 2006, p. 4, como citado en Bernal y Galindo, 2012, p. 175).

En este sentido, la institución educativa, a través de los conocimientos sociales y geográficos, debe desarrollar relaciones con los escolares a través el entorno más próximo, al promover la observación y la interpretación a diferentes escalas mediante un lenguaje cartográfico. Hay que potenciar en los discentes el pensamiento espacial con un enfoque integral y sistemático obteniendo las 
habilidades necesarias para interaccionar con el medio físico y social (Araya y Herrera, 2013).

PERSPECTIVA INTERNACIONAL SOBRE LA UTILIZACIÓN DE LOS MAPAS HISTÓRICOGEOGRÁFICOS EN LAS CLASES

Se han llevado a cabo diversas investigaciones en diferentes partes del mundo, por ejemplo, Hoyrup y Flonneau (1985) elaboran un cuaderno de cartografía con actividades para clase en Ligugé (Francia). Boardman, Carter y Sandfort (1986), lo recoge y trabaja en su manual Handbook for geography teachers en Sheffield (Reino Unido), o Doin de Almeida (2012) analiza la cartografía escolar desde São Paulo (Brasil). Entre los españoles destaca el cosmógrafo Santa Cruz (citado en Maroto y Piñeiro, 2006), para comentar que la Geografía se centraba en las escalas pequeñas, tales como, mapas del mundo, continentes o grandes regiones, mientras que la cartografía debía de encargarse de las representaciones detalladas, refiriéndose a vistas, pinturas, mapas de escala grande o planos de población.

Otro ejemplo, es el que lleva a cabo Rodrigo (2005 y 2006, como citado en Sebastiá y Tonda, 2015), al examinar las aplicaciones de los mapas en los libros de texto en la educación secundaria obligatoria en España y Brasil. Esta investigación se basa en dos clasificaciones de análisis, la primera hace referencia a habilidades de reconocer, situar y recordar; y la segunda, incluye destrezas en razonar, contrastar, indagar e interaccionar con los contenidos cartográficos.

Igualmente, en el sistema educativo costarricense, la enseñanza de la Geografía se asocia, tradicionalmente, a la de la Historia. Para que el estudiante logre un aprendizaje adecuado de la disciplina, es necesario un contacto directo con el entorno geográfico (paisaje geográfico) mediante el análisis de los fenómenos geográficos por medio de imágenes, mapas, gráficos: "En Costa Rica, la representación, por medio del uso del mapa, se constituye en el elemento fundamental del análisis geográfico" (Vargas, 2009, p. 77).
También en la Provincia de La Pampa, Argentina, los materiales curriculares del Ministerio de Cultura y Educación incorporan a la enseñanza de la Geografía y la Historia cuestiones relacionadas con la dimensión espacial y temporal de los procesos sociales. Se tienen en cuenta diversos factores: cómo se construyen y transforman los territorios, las intencionalidades de los diferentes actores sociales, el impacto de las cuestiones socio-culturales, entre otros. Este enfoque supone elaborar una propuesta de enseñanza $y$ aprendizaje que capacite a los educandos a entender el espacio geográfico como un proceso de construcción social sujeto a continuos cambios. Desde esta perspectiva, el manejo de técnicas de representación cartográfica, tradicionales e innovadoras, son necesarias para cualquier código de comunicación (Pombo et al., 2017).

Proyectar problemas históricos es una habilidad que se aprende $y$ que ha de explicitarse en la enseñanza (Arteaga y Camargo, 2014; Barton, 2008; Vansledright, 2014). La utilización de los mapas históricos en el aula favorece el desarrollo de una comprensión crítica y reflexiva sobre la aplicación didáctica de la cartografía como elemento de la cultura material escolar. En México, por ejemplo, "el espacio histórico se trabaja simultáneamente con Geografía e implica el uso de conocimientos cartográficos y el desarrollo de habilidades de localización y de interrelación de los elementos naturales y humanos" (Lima et ál., 2010, p. 10).

De acuerdo con Molina (como citado en Algranati et al., 2012), la cartografía es utilizada para explorar el entorno socio-territorial mediante la construcción de mapas. Se enseñan conocimientos que permiten al estudiantado un mejor conocimiento de su realidad creando espacios de reflexión y construyendo un lenguaje común de interpretación sobre las diferentes percepciones entre las comunidades.

En concreto, la cartografía social pedagógica (CSP) es un método de investigación que se utiliza con los discentes para comprender una problemática común que acontece en los territorios. A través de ejercicios prácticos de enseñanza-aprendizaje con los mapas se afianza 
la participación y la cooperación entre los escolares (Barragán y Amador, 2014).

Por ejemplo, trabajar con mapas en un entorno próximo desde diferentes perspectivas le proporciona al estudiantado una mayor comprensión de su realidad. "La cartografía local es convertida en un instrumento clave en la formación para el desarrollo desde el poder del lugar. La representación de lo concreto y lo real, permite descubrir el camino por el cual avanzar desde formas accesibles al ciudadano" (Rojas, 2000, p. 1).

La elaboración de este recurso contribuye a poseer una representación del espacio de forma significativa $y$ didáctica que permite a los docentes explicar el dinamismo espacial, la historia y la sociedad para que los escolares se orienten, localicen y asimilen un hecho o un determinado período de la historia, así como para el razonamiento respecto la evolución del espacio a lo largo del tiempo (Santaella, 1989).

\section{PROCEDIMIENTO METODOLÓGICO PARA EL ANÁLISIS DE LOS DATOS}

La metodología de análisis para el tratamiento de la información se ha centrado en las observaciones de los relatos de vida. Se concreta en la experiencia del estudiantado en sus clases de Historia. Cada relato (traducido en esta ocasión en una descripción) enuncia una lectura crítica de una situación determinada mediante la experiencia biográfica. Como investigación cualitativa, sus datos provienen de la vida cotidiana, del sentido común, de las explicaciones y las reconstrucciones que el alumnado considera para vivir cotidianamente (Ruiz, 2012).

La investigación se llevó a cabo con 258 estudiantes de secundaria en la Comunidad Autónoma de la Región de Murcia (España), con una edad de 17 años, elegidos de forma aleatoria. A través del recuerdo sobre su experiencia personal, se les preguntó cómo daban clase sus profesores de Historia Contemporánea a través del uso de unos medios específicos como son los mapas histórico-geográficos. La investigación se fundamenta en la forma en la que el estudiantado asimiló las clases impartidas de esta materia, con una mirada reflexiva hacia el pasado construida desde el presente. El tipo de memoria utilizada:

$\diamond \quad$ Trata de evocar un recuerdo de una temporalidad mínima de un año sobre una actividad concreta. Se refiere a la utilización de la Memoria a Largo Plazo.

$\diamond \quad$ Tiene un componente declarativo, ya que la tarea es narrar un suceso determinado que se hace consciente, aunque este se manifieste de manera constante a lo largo de un curso escolar.

$\diamond \quad$ Es episódica, porque se manifiestan tanto los componentes perceptivos como semánticos, asimismo, alude a una memoria explícita o consciente.

$\diamond \quad$ Hace referencia al alumnado y es un suceso de su experiencia personal, relacionándolo con la memoria autobiográfica.

\section{RESULTADOS}

La pregunta que se hizo al estudiantado, como se ha comentado anteriormente, estaba referida a la forma en la que sus profesores de Historia impartían clases habitualmente. Los resultados obtenidos se refieren a las aportaciones presentadas por los escolares sobre el uso que se hace de los mapas histórico-geográficos en las clase de Historia, las cuales se encuentran representadas en sub-apartados en la tabla 1.

El análisis de los datos se lleva a cabo con la LOGSE (Jefatura del Estado, 1990) debido a que es una Ley educativa que transfigura la organización escolar en España y que promueve una reforma de los contenidos educativos y actividades didácticas, además fue la primera ley que estableció un sistema descentralizado de enseñanza en España al asentir que las comunidades autónomas no solo tramitasen los centros educativos, sino que pudieran transcribir un porcentaje relevante de los contenidos curriculares. En concreto, en la etapa de Secundaria Obligatoria trata de ver cómo articular las áreas de conocimiento de las Ciencias Sociales, Geografía e Historia. Del mismo modo, la Ley 
Orgánica de Educación (LOE) (Jefatura del Estado, 2006) ha significado una evolución importante del currículo de secundaria. En la adquisición de la competencia Conocimiento y la interacción con el mundo físico se circunscriben aspectos como la percepción y el conocimiento del espacio físico en el que se interpreta la actividad de enseñanza. Obtienen especial notabilidad, los procedimientos de orientación, localización, observación e interpretación de los espacios y paisajes reales o representados. Los argumentos manejados han permitido incidir en las limitaciones y posibilidades de las disciplinas para efectuar una lectura reflexiva y metódica entre los docentes $y$ discentes en ambas leyes educativas tan imperantes en la historia de España.

En 1990, la Ley Orgánica de Ordenación General del Sistema Educativo estableció en diez años el período de obligatoriedad escolar y proporcionó un impulso y prestigio profesional y social a la formación profesional que permitiría finalmente equiparar a España con los países más avanzados de su entorno. Como consecuencia de esa voluntad expresada en la Ley, a finales del siglo Xx se había conseguido que todos los jóvenes españoles de ambos sexos asistiesen a los centros educativos al menos entre los seis y los dieciséis años y que muchos de ellos comenzasen antes su escolarización y la prolongasen después. Se había acortado así una distancia muy importante con los países de la Unión Europea, en la que España se había integrado en 1986 (Jefatura del Estado, 2006, p.5).

TABLA 1

APLICACIÓN DE LOS MAPAS HISTÓRICO-GEOGRÁFICOS EN EL AULA MURCIA, ESPAÑA, 2018

\begin{tabular}{ll}
\hline SUB-APARTADOS DE LAS RESPUESTAS & CANTIDAD \\
\hline Localizaciones espaciales & 44 \\
Reforzar las explicaciones & 96 \\
Realizar trabajos & 11 \\
Hacer más accesible el contenido & 16 \\
No se utilizan mapas por parte del profesor & 13 \\
Aportaciones y valoraciones recogidas sobre los mapas & 78 \\
\hline TOTAL DE APORTACIONES & 258 \\
\hline
\end{tabular}

Fuente: $\quad$ Elaboración propia, 2019.

Se especifica un estudio general referido a la preferencia que tienen los docentes cuando utilizan los mapas, en las interpretaciones formadas por el alumnado de secundaria, tanto en la Ley Orgánica de Educación (Jefatura del Estado, 2006) como en la Ley Orgánica General del Sistema Educativo (Jefatura del Estado, 1990) en España, mostrando ejemplos del número de respuestas ofrecidas como por la naturaleza de las mismas.
A) PREGUNTA: LOCALIZACIONES ESPACIALES (SITUAR LUGARES HISTÓRICOS, CIUDADES GEOGRÁFICAS, PAÍSES)

Ejemplos de respuestas del estudiantado (Jefatura del Estado, 2006):

135. Solía llevar mapa para situarnos en el lugar de los hechos.

69. Entraba a clase con el mapa, señalaba los países e iba preguntando. 
215. Aveces ponía mapas si el tema a dar lo requería pero no era muy frecuente, solamente puso mapas cuando estábamos dando la geografía de España.

83. Utilizaba mapas para situar las ciudades geográficas.

46. A veces señalaba en el mapa los países implicados en los acontecimientos.

Ejemplos de respuestas del estudiantado (Jefatura del Estado, 1990):

97. Señalaba en el mapa los lugares que aparecían en el tema.

55. Llevaba siempre el mapa en la mano y nos hacía identificar algún hecho de algún lugar en concreto.

110. Ilustraba sus clases con mapas para así situarnos los lugares donde se libraron las batallas, ciudades importantes $y$ otros muchos puntos de interés.

203. Con los mapas aprendí muy bien a localizar y ubicar lugares.

89. Alguna vez, mi profesor utilizaba mapas para intentar situarnos e indicarnos donde ocurrieron algunos hechos históricos.

TABLA 2

APORTACIONES DE LAS LOCALIZACIONES

ESPACIALES UTILIZADAS A TRAVÉS DE LOS MAPAS HISTÓRICO-GEOGRÁFICOS EN CLASE MURCIA, ESPAÑA, 2018

\begin{tabular}{cc}
\hline SISTEMA & CASOS \\
\hline LOE & 130 \\
LOGSE & 128 \\
\hline TOTAL & 258 \\
\hline
\end{tabular}

Fuente: $\quad$ Elaboración propia, 2019.

En la tabla 2 se destaca que las localizaciones espaciales por parte del docente son ligeramente más usadas en la LOE (Jefatura del Estado, 2006), según las respuestas de las personas encuestadas. La LOE (Jefatura del
Estado, 2006, art. 23e, p. 26) insiste entre sus objetivos para el alumnado de Educación Secundaria: "Desarrollar destrezas básicas en la utilización de las fuentes de información para, con sentido crítico, adquirir nuevos conocimientos (...)" y "Apreciar la creación artística y comprender el lenguaje de las distintas manifestaciones artísticas, utilizando diversos medios de expresión y representación" (Jefatura del Estado 2006, art. 23i, p. 27), de ahí su utilización en el aula. Igualmente, la Logse (Jefatura del Estado, 1990) destaca la diferenciación espacial (localización, distribución y organización), como resultado del estudio sincrónico de las sociedades y culturas en los procesos de evolución $y$ cambio en la experiencia humana.

B) PREGUNTA: REFORZAR LAS EXPLICACIONES (DIBUJAR, ILUSTRAR, MOSTRAR, COMPLEMENTAR MAPAS)

Ejemplos de respuestas del estudiantado (Jefatura del Estado, 2006):

91. Comentaba las fotos y mapas del libro.

127. La mayoría de las veces las explicaciones las ilustraba en distintos mapas. 263. Utilizaba mapas y comentarios de textos.

29. Habitualmente las explicaciones las hacia apoyándose en mapas.

Ejemplos de respuestas del estudiantado (Jefatura del Estado,1990):

66. Aveces traía mapas enormes para explicar las colonizaciones.

164. En ocasiones utilizaba mapas para explicar.

113. Utiliza mucho los mapas, siempre trabajando con ellos. Lo primero que hacía al llegar a clase era abrir el libro y desliar el mapa.

77. Usaba el mapa para complementar las explicaciones. 
TABLA 3

APORTACIONES DE LOS MAPAS HISTÓRICOGEOGRÁFICOS EN CLASE PARA REFORZAR LAS EXPLICACIONES MURCIA, ESPAÑA, 2018

\begin{tabular}{cc}
\hline SISTEMA & CASOS \\
\hline LOE & 147 \\
LOGSE & 111 \\
\hline TOTAL & 258 \\
\hline
\end{tabular}

Fuente: $\quad$ Elaboración propia, 2019.

En la tabla 3 se muestra que el docente para reforzar las explicaciones en la LOE hace mayor uso de los mapas que en el sistema educativo de la LOGSE, según las respuestas de las personas encuestadas. Uno de los objetivos principales de la LOE es "conocer, valorar y respetar los aspectos básicos de la cultura y la historia propias y de los demás, así como el patrimonio artístico y cultural" (Jefatura del Estado, 2006, art. 23j, p. 27). Del mismo modo, la LOGSE, busca una "la relación con el entorno social, económico y cultural del alumnado" y "El desarrollo de las capacidades creativas y de] espíritu crítico" (Jefatura del Estado, 1990, art. 2 j, $y$ art. 2d, p. 12).

C) PREGUNTA: REALIZAR TRABAJOS (HACER MAPAS)

Ejemplos de respuestas del estudiantado (Jefatura del Estado, 2006):

52. Uno de los mapas vistos en las clases prácticas nos lo preguntaba en el examen.

169. Si había mandado hacer mapas los recogía.

245. Siempre traía mapas y mandaba para hacer en casa.

80. La mayoría de las veces utilizábamos mapas para trabajar.

61. Hicimos muchos mapas y comentarios de texto.

Ejemplos de respuestas del estudiantado (Jefatura del Estado, 1990):

15. Hacíamos mapas históricos que nos facilitaban el estudio.
39. Mapas no vi muchos, porque no di nunca Geografía y en Historia de España hicimos algún mapa pero casi nada.

140. También hacíamos mapas.

TABLA 4

APORTACIONES SOBRE LOS MAPAS HISTÓRICOGEOGRÁFICOS

UTILIZADOS EN CLASE PARA HACER TRABAJOS MURCIA, ESPAÑA, 2018

\begin{tabular}{cc}
\hline SISTEMA & CASOS \\
\hline LOE & 143 \\
LOGSE & 115 \\
\hline TOTAL & 258 \\
\hline
\end{tabular}

Fuente: $\quad$ Elaboración propia, 2019.

En la tabla 4 se observa que en con la LOE el estudiantado realiza más trabajos con los mapas histórico-geográficos que en la LOGSE. Siguiendo los comentarios anteriormente expuestos y con las finalidades recogidas y establecidas en la legislación, la LOE incide en "Desarrollar el espíritu emprendedor y la confianza en sí mismo, la participación, el sentido crítico, la iniciativa personal y la capacidad para aprender a aprender, planificar, tomar decisiones y asumir responsabilidades" (Jefatura del Estado, 2006, art. 23g, p. 27) y así queda evidenciado en la práctica docente. Aunque en la LOGSE se señala que es necesaria "La adquisición de hábitos intelectuales y técnicas de trabajo, así como de conocimientos científicos, técnicos, humanísticos, históricos y estéticos" (Jefatura del Estado, 1990, art. 1c, p. 11).

D) PREGUNTA: HACER MÁS ACCESIBLE EL CONTENIDO (UTILIZACIÓN DE MAPAS)

Ejemplos de respuestas del estudiantado (Jefatura del Estado, 2006):

115. Repasábamos la lección ayudándonos con el mapa de clase.

230. Utilizaba mapas para situarnos.

53. Un día a la semana teníamos clase de mapas.

76. Alguna vez trajo un mapa (para que nos orientásemos). 
94. Gracias a los mapas resultaba más fácil la memorización del lugar donde sucedían los hechos que se estaban explicando en clase.

Ejemplos de respuestas del estudiantado (Jefatura del Estado, 1990):

201. Utilizaba diapositivas y mapas para señalarle a los alumnos algo importante del temario.

30. De vez en cuando repartía mapas y fotocopias para que las viésemos en casa.

41. Para que entendiéramos mejor las cosas, utilizaba mapas, documentos, transparencias, etc.

120. Cuando se trataba de Geografía (conquistas, colonialismo y expansionismo) colocaba un mapa en la pizarra para la mejor comprensión del tema.

68. Solía utilizar mapas para que nos pudiéramos orientar en el espacio.

TABLA 5

APORTACIONES DE LOS MAPAS HISTÓRICOGEOGRÁFICOS EN CLASE

PARA HACER MÁS ACCESIBLE EL CONTENIDO MURCIA, ESPAÑA, 2018

\begin{tabular}{cc}
\hline SISTEMA & CASOS \\
\hline LOE & 135 \\
LOGSE & 123 \\
\hline TOTAL & 258 \\
\hline
\end{tabular}

Fuente: $\quad$ Elaboración propia, 2019.

La tabla 5 refleja que para hacer más accesible el contenido no hay diferencias en la LOE y en la LOGSE, según las respuestas de las personas encuestadas. Como se ha comentado en las anteriores tablas, la finalidad de ambas leyes es "Utilizar con sentido crítico los distintos contenidos y fuentes de información, $y$ adquirir nuevos conocimientos con su propio esfuerzo" (Jefatura del Estado, 1990, art. 19c, p. 12).

E) PREGUNTA: NO SE UTILIZAN MAPAS POR PARTE DEL PROFESOR (REHÚSO Y DENIEGO HACIA LOS MAPAS)

Ejemplos de respuestas del estudiantado (Jefatura del Estado, 2006):
136. Muy pocas veces nos ponía mapas, o mejor dicho nunca.

250. Ponía vídeos en clase (no utilizaba mapas casi nunca) y películas como "tiempos modernos" o "el gran dictador", que a la vez que enseñaban divertían.

74. El material utilizado le parece insuficiente ya que podría haber utilizado mapas, transparencias o esquemas.

Ejemplos de respuestas del estudiantado (Jefatura del Estado, 1990):

41. Nunca utilizaba mapas ni transparencias. 84. Pensaba que el material complementario (vídeo, transparencias, mapa, etc.) era una pérdida de tiempo.

107. Mi profesor no utiliza mapas, transparencias o diapositivas cuando explica.

TABLA 6

APORTACIONES DE QUE NO SE UTILIZAN MAPAS HISTÓRICO-GEOGRÁFICOS

EN CLASE POR PARTE DEL PROFESORADO MURCIA, ESPAÑA, 2018

\begin{tabular}{cc}
\hline SISTEMA & CASOS \\
\hline LOE & 83 \\
LOGSE & 175 \\
\hline TOTAL & 258 \\
\hline
\end{tabular}

Fuente: $\quad$ Elaboración propia, 2019.

La Tabla 6 indica una diferencia de casi el triple en la actitud del docente ante los mapas, según las respuestas de las personas encuestadas, utilizándose estos bastantes menos en la LOGSE que la LOE.

F) PREGUNTA: APORTACIONES Y VALORACIONES RECOGIDAS SOBRE LOS MAPAS (IMPORTANCIA DE LOS MAPAS: UTILIZABA MAPAS Y SE AYUDABA DE ELLOS; FRECUENCIA Y CANTIDAD DE USO DE LOS MAPAS: A VECES, POCO, RARA VEZ, MUCHOS).

Ejemplos de respuestas del estudiantado (Jefatura del Estado, 2006):

95. Nos ponía muchos mapas.

109. Es frecuente que utilice mapas.

33. Generalmente llevaba con ella un mapa que colgaba en la pizarra. 
244. Algunas veces ponía mapas en la pizarra que adornaban ya que no se movía a por ellos.

Ejemplos de respuestas del estudiantado (Jefatura del Estado, 1990):

185. Siempre llevaba un mapa y lo colgaba. 48. Al entrar a clase traía muy frecuentemente los mapas.

79. Ponía mapas que cubrían toda la pizarra.

25. Entraba a clase cargada de mapas y con el retroproyector.

TABLA 7

APORTACIONES Y VALORACIONES DE LOS MAPAS HISTÓRICO-GEOGRÁFICOS EN CLASE MURCIA, ESPAÑA, 2018

\begin{tabular}{cc}
\hline SISTEMA & CASOS \\
\hline LOE & 144 \\
LOGSE & 114 \\
\hline TOTAL & 258 \\
\hline
\end{tabular}

Fuente: Elaboración propia.

En la tabla 7 se detalla que en la LOE se da más importancia a los mapas que en la LOGSE. En esta ley se destaca una diferencia de casi el doble en las aportaciones en cuanto a la frecuencia y cantidad de uso de los mapas según las respuestas de las personas encuestadas, aun así se recogen más aportaciones en la LOE.

\section{CONCLUSIONES}

El procedimiento empleado por el docente para fomentar la enseñanza-aprendizaje de forma significativa debe estar encauzado a través de estrategias que incluyan al estudiantado en su contexto sociocultural, a través de prácticas cotidianas, próximas, destacadas en su cultura, apoyadas en métodos de interacción social. De esta manera, se evitaría el aprendizaje memorístico, mecánico, cuantificado, instrumentalizado con diferentes técnicas y recursos educativos, como el empleo en el aula de los mapas histórico-geográficos para adquirir un dominio del temario centrado en la formación de actitudes críticas y el compromiso social (Sansevero et al., 2006).

La interpretación y elaboración de mapas son conocimientos ineludibles en la formación de los discentes para conocer la realidad que les rodea en la sociedad de la que forman parte. Manifiestan la precisión de ofrecer información espacial. A través de representaciones gráficas se proporciona un mejor entendimiento de la localización de objetos, conceptos, fenómenos, procesos o acontecimientos en el territorio $(\mathrm{Ca}-$ talá, 2016). "La educación como práctica socializa al individuo con el entorno; de tal manera que sus contenidos y la forma de concebir el mundo se transforma en elementos esenciales en el imaginario colectivo" (como citado en Agüero, 2014, p. 182).

En esta investigación, las apreciaciones que se consideran para la utilización de los mapas histórico-geográficos para las localizaciones espaciales son un factor relativo de referencia del profesorado en clase, opciones que muestran una clara preferencia para apoyar las explicaciones. Los profesores ilustraban sus clases con mapas para situarlos en lugares donde se libraron batallas, ciudades importantes y otros puntos de interés.

Cuando se trataba de Geografía (conquistas, colonialismo y expansionismo) colocaban mapas en la pizarra para la mejor comprensión de los temas explicados. Las localizaciones espaciales por parte del docente son ligeramente más usadas en la LOE que en la LOGSE. Igualmente, el docente para reforzar las explicaciones en la LOE hace mayor uso de los mapas que en el sistema educativo de la LOGSE, según las respuestas de las personas encuestadas. También se observa que en con la LOE el estudiantado realiza más trabajos con los mapas histórico-geográficos que en la LOGSE.

Sin embargo, se refleja que para hacer más accesible el contenido no hay muchas diferencias en ambos sistemas educativos (Jefatura del Estado, 1990; 2006). Del mismo, se indica una diferencia de casi el triple en la actitud del docente ante los mapas, utilizándose menos en la LOGSE que la LOE.

Se concluye que en la LOE se la da más importancia a los mapas que en la LOGSE. La escuela 
es el espacio en el que se perciben e integran las vivencias personales y colectivas de la mayoría de los educandos, se adquiere la mayor cantidad de conocimientos, se desarrollan actividades para fortalecer habilidades emocionales, capacidades cognoscitivas (atención, percepción, memoria, resolución de problemas, comprensión, etc.), psicomotrices y actitudinales del alumnado, que explican el mundo a partir de los procesos dinámicos que entrelazan la perspectiva social, cultural y educativa (Cárdenas-Pérez y TroncosoÁvila, 2014).

Los contenidos conceptuales son muy semejantes, la LOGSE establece y contempla los diferentes elementos que especifican una explicación geográfica utilizando para ello un vocabulario proporcionado y la LOE matiza los elementos básicos de la organización del territorio, utilizando conceptos y destrezas específicamente geográficas para analizar e interpretar un determinado fenómeno o situación territorial, valorando los múltiples factores que intervienen, utilizando en su descripción y explicación la terminología apropiada. El objetivo número 8 de la LOE explica la posición de España en un mundo interrelacionado, en el que coexisten procesos de uniformización de la economía y de desigualdad socioeconómica, no se propone en la LOGSE y solo parcialmente en el número 4 de la LOE.

El desarrollo de competencias y habilidades de conocimientos cartográficos se tienen en consideración en los distintos niveles del currículo, estos saberes son parte integrante de la enseñanza escolar. Es ineludible implantar los conceptos cartográficos en el aula de manera gradual y sistemática atendiendo a las necesidades del estudiantado en función de sus capacidades metacognitivas $y$ de aprendizaje.

\section{REFERENCIAS}

Agüero, J. (2014). Al progreso en la formación de ciudadanos eficientes: educación y sociedad en Costa Rica en 1930. Revista Ciencias Sociales, (145), 181-194.

Algranati, A., Bruno, D. y Lotti, A. (2012). Mapear actores, relaciones y territorios. Una herramienta para el análisis del escenario social. Cuadernos de Cátedra, (3), 1-22.
Andreozzi, G. y Benedetti, G. M. (6-8 de octubre de 2005). Aprender geografía desde la foto aérea. II Congreso de Didáctica de las Ciencias Sociales. Desafíos y nuevas construcciones en la enseñanza de las Ciencias Sociales. Necochea, Buenos Aires, Argentina.

Araya, F. y Herrera, Y. (2013). Estrategias docentes para el desarrollo de habilidades de pensamiento espacial en República Dominicana y Chile. Revista Geográfica de Valparaíso, (47), 27-41.

Araya, I. y Pacheco X. (2008). Mapas cognitivos. Aprendizajes desde la vivencia espacial. Revista Geográfica de América Central, (42), 11-29.

Arteaga, B. y Camargo, S. (2014). Educación histórica: una propuesta para el desarrollo del pensamiento histórico en el plan de estudios de 2012 para la formación de maestros de Educación Básica. Tempo e Argumento, Florianópolis, 6 (13), 110-139.

Barragán, D. F. y Amador, J. C. (2014). La cartografía social-pedagógica: Una oportunidad para producir conocimiento y repensar la educación. Itinerario Educativo, (64), 127-141.

Barton, K. (2008). Research on student's ideas about history. En L. Levstik y C. Thyson (Eds.), Handbook of research on social studies education (pp.239-258). Routledge.

Bernal, R. M. y Galindo, D.F. (2012). Cartografía social y sistemas de información geográfica. Una nueva experiencia en la educación. Revista digital del Grupo de Estudios sobre Geografía y Análisis Espacial con Sistemas de Información Geográfica (GESIG), (4), 169-186.

Boardman, D., Carter, D. y Sandford, H. (1986). Maps and Mapwork. En D. Boardman (Ed.), Handbook for geography teachers (pp. 123-148). Geographical Association.

Buisseret, D. (2004). La revolución cartográfica en Europa. Paidós.

Carrera, C., del Canto, C., Gutiérrez J., Méndez, R. y Pérez, M. (1993). Trabajos Prácticos de geografía humana. Editorial Síntesis. 
Cárdenas-Pérez, R. E. y Troncoso-Ávila, A. (2014). Importancia de las artes visuales en la educación: un desafío para la formación docente. Revista Electrónica Educare, 18 (3), 191-202.

Catalá, R. (2016). Explicando el mundo en el que vivimos con mapas: Propuesta de aprendizaje cartográfico. Geógrafos. Revista digital para estudiantes de Geografía y Ciencias Sociales, 7 (89), 171-206.

Clary, M. (1995). Els models gràfics en la construcció dels concepts geogràphics. Revista de Documents dAnàlisis Geogràfica, 27, 17-36.

Crespo, A. y Fernández, A. (2011). ¿Cartografía antigua o Cartografía histórica? Estudios Geográficos, 72 (271), 403-420.

Delgado, E. (2002). El mapa: importante medio de apoyo para la enseñanza de la historia. Revista Mexicana de Investigación Educativa, 7 (5), 331-357.

Doin de Almeida, R. (Coord.). (2012). Cartografia escolar. Editora Contexto.

Harley, B. (2005). La nueva naturaleza de los mapas. Ensayos sobre la historia de la cartografía. Fondo de Cultura Económica.

Hernando, A. (2000). La historia de la cartografía de América: entre la exaltación y la concienciación. En P. García Jordán (Coord.), Estrategias de poder en América Latina: Estratègies de poder a Amèrica Llatina (pp.25-44). Encuentro Debate América Latina Ayer y Hoy.

Hoyrup, E. y Flonneau, M. (1985). Cartographie: cahier d activités. Nathan.

Jefatura del Estado. (3 de octubre de 1990). Ley Orgánica Ordenación General del Sistema Educativo (LOGSE). Boletín Oficial del Estado, n ${ }^{\circ} 238$.

Jefatura del Estado. (3 de mayo de 2006). Ley Orgánica de Educación (LOE). Boletín Oficial del Estado, $n^{\circ} 106$.

Jerez, O. (2006). El lenguaje cartográfico como instrumento para la enseñanza de una geografía crítica y para la educación ambiental. En M. J. Marrón., M. J. Sánchez, L. y O. Jerez (Eds.), Cultura geográfica y educación ciudadana (pp.483-502). Grupo de Didáctica de la Geografía (AGE), Asociaçao de Profesores de Geografía de Portugal y Universidad de Castilla La Mancha.

Lima, L., Bonilla, B. y Arista, V. (2010). La enseñanza de la Historia en la escuela Mexicana. Proyecto Clío, (36), 1-16.

Luque, R. M. (2011). El uso de la cartografía y la imagen digital como recurso didáctico en la Enseñanza Secundaria. Algunas precisiones en torno a Google Earth. Boletín de la Asociación de Geógrafos Españoles, (55), 183-210.

Maroto, V. y Piñeiro, M. (2006). Aspectos de la Ciencia aplicada en la España del Siglo de Oro. Junta de Castilla y León.

Parellada, C. A. (2017). Los mapas históricos como instrumentos para la enseñanza de la historia. Tempo e Argumento, Florianópolis, 9 (21), 312-337.

Perea, R. y Mayor, J. C. (2014). La cartografía como instrumento de comunicación en la planificación del espacio geográfico. Entorno Geográfico, (10), 180-193.

Piñeiro, R. (2003). Innovación en didáctica de la geografía. En M. J. Marrón, C. Moraleda y H. Rodríguez (Coords.), La enseñanza de la geografía ante las nuevas demandas sociales (pp. 344-350). Grupo de Didáctica de la Geografía (AGE), Universidad de Castilla-La Mancha (UCLM).

Pombo, D., Martínez, Ma . C. y García, M.C. (2017). Las Tecnologías de Información Geográfica (TIG) y la Historia por medio de la resolución de problemas en la Escuela Secundaria. Revista del Departamento de Geografía, (8), 191-206.

Quinquer, D. (1997). Estrategias de enseñanza: los métodos interactivos. En P. Benejam y J. Pagès. Enseñar y aprender ciencias sociales, geografía e historia en la educación secundaria (pp. 97-119). Universitat de Barcelona, ICE / Horsori.

Raisz, E. (1985). Cartografía General. Omega.

Ramaswamy, S. (2001). Maps and mother goddesses in modern India. Imago Mundi, $53,97-114$. 
Real Academia Española. (2016). Diccionario de la lengua española (23. ad.). Espasa.

Rojas, A. (2000). Educación como Continuo Humano. Principio del Desarrollo de la Educación Bolivariana. Grupo Editorial Arte Estilo, S.A.

Ruiz, J. I. (2012). Historias de vida. Metodología de la Investigación Cualitativa. Universidad de Deusto.

Sansevero, I., Lúquez, P. y Fernández, O. (2006). Estrategias de aprendizaje significativo para la interiorización de valores de identidad nacional en la Educación Básica. Laurus, 12 (22), 273-300.

Santaella, R. (1989). La Dinámica del Espacio en la Cuenca del Lago de Maracaibo 1873- 1940. Facultad de Ciencias Económicas y Sociales (FacEs), Universidad Central de Venezuela.

Sebastiá, R. y Tonda, E. M. (2015). El concepto $y$ representación del espacio geográfico en la enseñanza de la Geografía en los niveles educativos no universitarios. Análisis bibliométrico. En J. De la Riva, P. Ibarra, R. Montorio y M. Rodrígues (Eds.). Análisis espacial y representación geográfica: innovación y aplicación (pp.1505-1514). Universidad de Zaragoza.

Souto, X.M. (1998). Didáctica de la Geografía. Conocimiento del medio y problemas sociales. Serbal.

Vansledright, B. (2014). Assessing historical thinking and understanding: Innovate designs for new standards. Routledge.

Vargas, G. (2009). Didáctica de la Geografía y su aplicación a la enseñanza de la Geografía en el tercer ciclo y la enseñanza diversificada de Costa Rica. Educación, 33(1), 75-112.

Fecha de ingreso: $14 / 02 / 2019$ Fecha de aprobación: 16/01/2020 
